\title{
Prone Ventilation in a 27 Week Pregnant Woman with COVID-19 Severe ARDS
}

\author{
Luigi Barile ${ }^{1, *}$, Martina Cerrano ${ }^{1}$, Alessandro Locatelli ${ }^{1}$, \\ Andrea Puppo ${ }^{2}$, Anna F. Signorile ${ }^{2}$, Nicoletta Barzaghi ${ }^{1}$
}

\author{
${ }^{1}$ Department of Anesthesia and \\ Intensive Care, Azienda Ospedaliera \\ Santa Croce e Carle, Via Michele Coppino \\ 26, 12100, Cuneo, Italy \\ ${ }^{2}$ Department of Obstetrics and \\ Gynecology, Azienda Ospedaliera Santa \\ Croce e Carle, Via Michele Coppino 26, \\ 12100, Cuneo, Italy

\section{*Correspondence} \\ luigi.barile85@hotmail.it \\ (Luigi Barile)
}

\begin{abstract}
Pregnant women are more sensitive to respiratory pathogens due to the physiological changes related to pregnancy with an increase in morbidity and mortality. Pregnancy and childbirth do not seem to aggravate the course of symptoms of COVID-19 pneumonia. However, reports on optimal management of severe COVID-19-related ARDS during pregnancy are still lacking. To our knowledge only two case reports describe prone ventilation in pregnant women with severe ARDS, no one related to COVID-19. We report the case of a COVID-19 related severe ARDS in a 48-year-old woman in the last trimester of pregnancy. The patient required intensive care hospitalization for 20 days and invasive mechanical ventilation for 15 days. Pronation maneuver during mechanical ventilation relieved hypoxia and prevented mother and fetus damages, thus avoiding an urgent cesarean section and a premature birth. The patient was successfully discharged from the hospital without maternal and fetal sequelae. In our experience prone ventilation can be safely used to improve respiratory gas exchanges in the last trimester of pregnancy in case of severe ARDS.
\end{abstract}

\section{Keywords}

COVID-19, Respiratory distress syndrome, Adult, Pronation, Pregnancy, Respiration, Artificial

\section{Introduction}

Viral pneumonia occurring during pregnancy is associated with increased morbidity and mortality [1]. Pregnant women are more susceptible to respiratory pathogens because of the pregnancy-related physiological changes [2]. Pregnancy does not seem to aggravate the course of symptoms and clinical characteristics of COVID19 pneumonia, and the first reports on COVID-19 epidemiology data show an approximately superimposable rate of intensive care unit (ICU) hospitalization [3, 4]. However, optimal management in severe COVID-19 related pneumonia during pregnancy is still debated. After obtaining written informed consent and the ethics committee approval (Comitato Etico Interaziendale, Cuneo, Italy), we report the case of a 28 -week pregnant woman with COVID-19 related ARDS.

\section{Case report}

On 27th of March 2019 a 48-years-old, $27+4$ weeks gravida 2, para 0 african woman was admitted to our hospital for severe respiratory failure. She was subjected to chest x-ray suggestive for COVID-19 pneumonia (Fig. 1), which was then confirmed by a positive nasal swab. Medical history included hypertension; obesity (BMI 30); sicklecell trait; stillbirth at 24 weeks. Pregnancy checks showed maternal and fetal wellness. A review of symptoms was significant for asthenia, nausea and vomiting. She was afebrile, with $85 \%$ oxygen saturation in air, respiratory rate (RR) $>20$ breaths/minute; heart rate of 95 beats/minute and blood pressure within normal range. After a non-invasive pressure support ventilation trial failure, the patient was admitted in ICU; ventilation parameters are shown in Table 1.

Upon multidisciplinary discussion with the Obstetrics/Gynecologists (Ob/Gyn) team, we decided to intervene with a cesarean-section (CS) in case of signs 
TA B L E 1. Type of ventilation or oxygen support and arterial blood gas analysis data during intensive care unit stay.

\begin{tabular}{|c|c|c|c|c|c|c|c|c|}
\hline & $\begin{array}{c}\text { ICU } \\
\text { admission }\end{array}$ & $\begin{array}{c}\text { First day } \\
\text { in ICU }\end{array}$ & $\begin{array}{l}\text { 3th day in } \\
\text { ICU }\end{array}$ & $\begin{array}{c}6 \text { hours after } \\
\text { prone } \\
\text { ventilation }\end{array}$ & $\begin{array}{c}\text { First day after } \\
\text { prone } \\
\text { ventilation }\end{array}$ & $\begin{array}{l}\text { 5th day after } \\
\text { prone } \\
\text { ventilation }\end{array}$ & $\begin{array}{c}\text { 14th day after } \\
\text { prone } \\
\text { ventilation }\end{array}$ & $\begin{array}{c}\text { Day of } \\
\text { discharge }\end{array}$ \\
\hline $\begin{array}{l}\text { Type of ventila- } \\
\text { tion or oxygen } \\
\text { support }\end{array}$ & $\begin{array}{c}\text { NIVP } \\
\text { with } \\
\text { helmet }\end{array}$ & $\mathrm{VCV}$ & $\mathrm{VCV}$ & $\begin{array}{l}\text { VCV prone } \\
\text { position }\end{array}$ & $\mathrm{VCV}$ & PSV & $\begin{array}{l}\mathrm{O}_{2} \text { nasal } \\
\text { cannulas }\end{array}$ & - \\
\hline $\begin{array}{l}\text { TV or PS (mL or } \\
\left.\mathrm{cmH}_{2} \mathrm{O}\right)\end{array}$ & 8 & 520 & 520 & 520 & 530 & 20 & - & - \\
\hline RR & - & 20 & 20 & 20 & 20 & - & - & - \\
\hline $\mathrm{PEEP}\left(\mathrm{cmH}_{2} \mathrm{O}\right)$ & 10 & 10 & 10 & 10 & 12 & 12 & - & - \\
\hline $\mathrm{FiO}_{2}$ & 0.9 & 0.8 & 0.8 & 0.7 & 0.7 & 0.7 & 0.35 & 0.21 \\
\hline $\mathrm{Dp}\left(\mathrm{cmH}_{2} \mathrm{O}\right)$ & - & 15 & 12 & 12 & 13 & - & - & - \\
\hline $\begin{array}{l}\mathrm{Cs} \\
\left(\mathrm{mL} / \mathrm{cmH}_{2} \mathrm{O}\right)\end{array}$ & - & 34 & 40 & 46 & 41 & - & - & - \\
\hline $\mathrm{PaO}_{2}(\mathrm{mmHg})$ & 63.6 & 86.5 & 59.9 & 156 & 99.9 & 182 & 93.2 & 124 \\
\hline $\mathrm{PaCO}_{2}(\mathrm{mmHg})$ & 31.5 & 42.2 & 42.6 & 41.9 & 41.4 & 42.8 & 32.5 & 32.8 \\
\hline $\mathrm{PaO}_{2} / \mathrm{FiO}_{2}$ & 70 & 108 & 66.6 & 222 & 143 & 243 & 207 & 443 \\
\hline $\mathrm{SpO}_{2}$ & 91.9 & 93.5 & 91.3 & 99.3 & 97.7 & 99.4 & 93.2 & 99 \\
\hline
\end{tabular}

$T$ v, tidal volume; PS, pressure support; RR, respiratory rate; PEEP, positive end-espiratory pressure; FiO2, fraxion of inspired oxygen; Dp, driving pressure; Cs, static compliance; PaO2, partial pressure of oxygen in arterial blood sample; $\mathrm{PaCO}$, partial pressure of carbon dioxide in arterial blood sample; SpO2, blood oxygen saturation; NIVP, non invasive pressure support ventilation; VCV, volume controlled ventilation; PSV, pressure support ventilation.

of severe hypoxia ( $\mathrm{PaO} 2 / \mathrm{FiO} 2<100$ for $>3$ hours), hemodynamic instability (median artery pressure (MAP) $<65 \mathrm{mmHg}$ or the need of inotropic/vasoconstrictor drugs) or a life-threatening worsening of maternal conditions.

Fetal wellbeing was assessed by daily cardiotocogram and periodic obstetric ultrasounds to ensure regular fetal growth, as recommended [2]; in the event of a premature birth, we administered betamethasone $(12 \mathrm{mg}$ iv once for two days); no more corticosteroids were administered during ICU stay.

Sedation was achieved with a combination of remifentanil (50-100 ng/kg/min) and midazolam (3-8 mg/h) guided by electroencephalogram parameters in order to use the lowest dose of sedative drugs; cisatracurium was used as a miorelaxant agent $(2-3 \mathrm{mcg} / \mathrm{kg} / \mathrm{min})$. The patient was kept in a supine position alternating left lateral decubitus. We maintained a tidal volume of $6-8 \mathrm{ml} / \mathrm{kg}$ (ideal body weight correct for gestational age), a plateau pressure below 30 $\mathrm{cmH} 2 \mathrm{O}$ and a driving pressure below $15 \mathrm{cmH} 2 \mathrm{O}$; PEEP and $\mathrm{FiO} 2$ were set in order to achieve a $\mathrm{PaO} 2>70 \mathrm{mmHg}$ and $\mathrm{RR}$ set to keep PaCO2 in normal range (Table 1). Hemodynamic parameters were monitored with a non-invasive advanced hemodynamic monitor. Bed-side ultrasounds were used for daily monitoring of the lungs. According to our institutional COVID-19 treatment protocol, therapeutic dose of enoxaparin was given to prevent thromboembolic complications; serial evaluation of tromboelastography and anti-Xa factor dosage were monitored. Hydroxychloro- quine (400 mg bid for the first two days and $200 \mathrm{mg}$ bid for other three days) and Darunavir+Cobicistat $(800+150$ $\mathrm{mg}$ once for five days) were also started. Notably, all the given drugs are reported to have a good safety profile in pregnancy $[2,3]$.

On the third day of ventilation gas exchange worsened; we started a trial of prone ventilation as rescue therapy. The patient was prone positioned with anti-decubitus head ring-rolls protection and foam dressings placed under the shoulders, thighs and legs. In order to avoid abdominal compression the anti-decubitus mattress was deflated at the abdomen level. Arms were aligned with the body keeping the shoulders in a neutral position; the mild antiTrendelenburg position was chosen to reduce face and head swelling. Prone ventilation lasted 16 hours without hemodynamic derangements. Umbilical artery doppler monitoring ensured fetal well-being before and after prone positioning.

In the following days $\mathrm{PaO} 2 / \mathrm{FiO} 2$ values remained above 100. Seven days after ICU admission, bronchoalveolar lavage (BAL) cultures tested positive for Klebsiella pneumoniae sensitive to piperacilline/tazobactam which was administered for 10 days, obtaining a negative BAL result at the end of antibiotic course. Body temperature over $38.5^{\circ} \mathrm{C}$ where solved with paracetamol; no fever was detected after the tenth day of hospitalization.

Fifteen days after ICU admission the patient was able to breathe spontaneously with a decreasing need of oxygen 


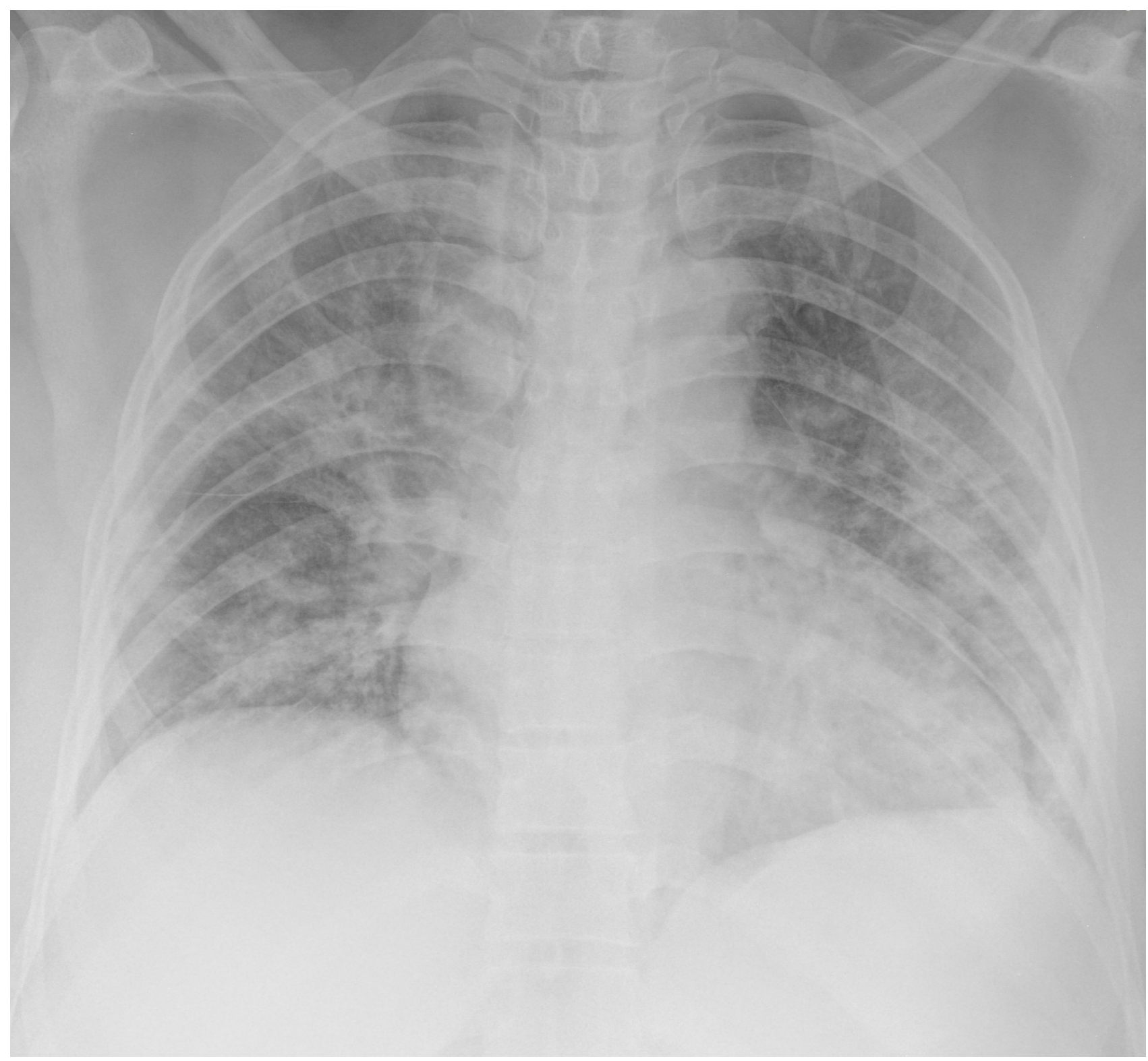

F I G U R E 1. Chest x-ray at emergency department admission.

supplementation. On the 19th and 20th day of hospitalization she tested negative for COVID-19 on two RNA PCR. Accordingly, she was transferred to the Ob/Gyn ward and at 31 weeks pregnant the patient was discharged at home and scheduled for periodic obstetrics checks.

\section{Discussion}

ARDS remained the most common cause of ICU maternal and fetal morbidity and mortality. Di Mascio et al. reviewed data of 41 COVID-19 positive pregnant patients, reporting $41.1 \%$ preterm birth $<37$ weeks, $18.8 \%$ premature rupture of membranes, $13.6 \%$ preeclampsia, $91.1 \%$ cesarean delivery; $2.4 \%$ stillbirths, $10 \%$ hospitalization in neonatal intensive care and $2.4 \%$ neonatal death [5]. Recent case series suggest mothers with severe COVID-19 pneumonia in the third trimester usually need a premature CS upon hospital admission [3], but it is not yet clear whether management of the mother's respiratory disease is improved by childbirth.

Prone ventilation is often used as a rescue therapy to treat severe ARDS; it allows greater alveolar recruitment of the dorsal lung areas. Benefits are an augmented ventilationperfusion ratio, a decreased compression by the heart of posterior and medial lung areas, a reduction in hypoxic vasoconstriction and an improvement in cardiac output [6]. In our opinion pregnant patients may have further advantages: diaphragm is moved caudally decreasing the compression of the posterior and caudal pulmonary parenchyma, as described in obese patients [7]; major vessels are less compressed from the uterus if the abdomen is left unsupported.

Despite benefits of pronation in severe ARDS have been widely established [6], data on its use in pregnant patients are anecdotal. Oliveira et al. studied the prone position in awake and spontaneously air breathing patients in 20- 
37 week gravida. Prone position was considered safe, comfortable and advantageous to improve oxygen saturation [8], however short duration of pronation was the main limitation. To our knowledge only two case reports describe prone ventilation in pregnant women with severe ARDS. Kenn et al. reported the case of a pregnant woman at 34 weeks of gestation, treated for an ARDS following blunt chest trauma. She obtained benefits from a single prone ventilation cycle [9]. The group of Sukhen describe a 31 weeks of gestation pregnant woman with a refractory ARDS due to H1N1 influenza. Three consecutive cycles of pronation were needed to obtain an improvement in oxygenation [10].

Compared to these cases, our patient is older, some weeks younger in gestational age, and overweight, which makes pronation challenging. During pronation maneuver our ICU staff was extremely careful in positioning and we carried out fetal monitoring before and after positioning.

Prone ventilation may be considered to improve respiratory gas exchanges in pregnant women with severe ARDS. Although it was performed only once, prone position allowed us to avoid protracted maternal hypoxia resulting in early premature birth.

\section{ACKNOWLEDGEMENTS}

We would like to thank the Intensive Care Unit staff of the Azienda Ospedaliera Santa Croce e Carle (Cuneo).

\section{CONFLICT OF INTEREST}

The authors declare no conflict of interest.

\section{FUNDING INFORMATION}

This research did not receive any specific grant from funding agencies in the public, commercial, or not-for-profit sectors.

\section{REFERENCES}

[1] Mehta N, Chen K, Hardy E, et al. Respiratory disease in pregnancy. Best Practice \& Research Clinical Obstetrics and Gynaecology. 2015;29:598-611.

[2] Bhatia PK, Biyani G, Mohammed S, et al. Acute respiratory failure and mechanical ventilation in pregnant patient: A narrative review of literature. J Anaesthesiol Clin Pharmacol. 2016;32:431-439.

[3] Liu H, Wanga LL, Zhaoa SJ, et al. Why are pregnant women susceptible to COVID-19? An immunological viewpoint. J Reprod Immunol. 2020;139:103122.

[4] Liu D, Li L, Wu X, et al. Pregnancy and Perinatal Outcomes of Women With Coronavirus Disease (COVID-19) Pneumonia: A Preliminary Analysis. AJR Am J Roentgenol. 2020;18:1-6.

[5] Di Mascio D, Khalil A, Saccone G, et al. Outcome of Coronavirus spectrum infections (SARS, MERS, COVID 1 -19) during pregnancy: a systematic review and meta-analysis. Am J Obstet Gynecol MFM. 2020;2:100107.

[6] Pelosi P, Brazzi L, Gattinoni L. Prone position in acute respiratory distress syndrome. Eur Respir J. 2002;20:1017-28.

[7] Pelosi P, Croci M, Calappi E, et al. Prone positioning improves pulmonary function in obese patients during general anesthesia. Anesth Analg. 1996;83:578-83.

[8] Oliveira C, Lopes MAB, Rodrigues AS, et al. Influence of the prone position on a stretcher for pregnant women on maternal and fetal hemodynamic parameters and comfort in pregnancy. Clinics (Sao Paulo). 2017;72:325-332.

[9] Kenn S, Weber-Carstens S, Weizsaecker K, et al. Prone positioning for ARDS following blunt chest trauma in late pregnancy. Int J Obstet Anesth. 2009;18:268-71.

[10] Samanta S, Samanta S, Wig J, et al. How safe is the prone position in acute respiratory distress syndrome at late pregnancy? Am J Emerg Med. 2014;32:687.e1-3.

How to cite this article: Luigi Barile, Martina Cerrano, Alessandro Locatelli, Andrea Puppo, Anna F. Signorile, Nicoletta Barzaghi. Prone Ventilation in a 27 Week Pregnant Woman with COVID-19 Severe ARDS. Signa Vitae. 2020;16(1):199-202. doi:10.22514/sv.2020.16.0028. 